University of Wollongong

Research Online

Faculty of Engineering - Papers (Archive)

Faculty of Engineering and Information

Sciences

$1-1-2012$

\title{
Terahertz photon mixing effect in gapped graphene
}

Yee Sin Ang

University of Wollongong, ysa190@uowmail.edu.au

Shareef Sultan

University of Wollongong

Asya Tawfiq

University of Wollongong, aat495@uowmail.edu.au

Juncheng Cao

Shanghai Institute of Microsystem and Information Technology

Chao Zhang

University of Wollongong, czhang@uow.edu.au

Follow this and additional works at: https://ro.uow.edu.au/engpapers

Part of the Engineering Commons

https://ro.uow.edu.au/engpapers/4891

\section{Recommended Citation}

Ang, Yee Sin; Sultan, Shareef; Tawfiq, Asya; Cao, Juncheng; and Zhang, Chao: Terahertz photon mixing effect in gapped graphene 2012, 816-824.

https://ro.uow.edu.au/engpapers/4891

Research Online is the open access institutional repository for the University of Wollongong. For further information contact the UOW Library: research-pubs@uow.edu.au 


\title{
Terahertz Photon Mixing Effect in Gapped Graphene
}

\author{
Yee Sin Ang, Shareef Al Tikrity, Asya Tawfiq, Juncheng Cao, Chao Zhang*
}

School of Engineering Physics, University of Wollongong, New South Wales 2522,

\section{Australia}

*Email: czhang@uow.edu.au

\begin{abstract}
We theoretically calculate the nonlinear terahertz waves mixing effect in doped graphene with a finite bandgap. The temperature dependence of the nonlinear intraband optical response at bandgap opening of few tens of meV are investigated. When the external electric field is weak, a moderate level of bandgap opening is found to slightly enhance the nonlinear optical response. The optical response is however significantly altered under strong-field condition. The strong-field nonlinear optical conductivity exhibits two distinct response 'hot spot': (i) low temperature with large bandgap and (ii) high temperature with small bandgap. The electric field required for the nonlinear response to dominate over the linear response is typically in the order of $10^{4} \mathrm{~V} / \mathrm{cm}$. This value increases rapidly by a factor of 10 in large bandgap and high temperature regimes. Our results suggest that photon mixing effect in gapped graphene is strongly gapped dependent and hence the bandgap opening has to be carefully engineered in order to optimize the photon mixing effect in gapped graphene.
\end{abstract}


Graphene is a hexagonal lattice structure made up of entirely carbon atom. It was first isolated in 2004 [1] and has attracted immense research in recent years. Electronically, pure graphene is a gapless semiconductor with its conduction band touches the valence point at the $\mathrm{K}$ point, or typically known as the 'Dirac point', of its Brillouin zone. The quasiparticle around the Dirac point behaves like a massless ultra-relativistic particles and this peculiar quasiparticle dynamics has given rise to many exotic physical properties [2-10]. In terms of electronic applications, the absence of a bandgap in its electronic band structure is usually considered the Achilles' heel of graphene. A gapless graphenebased transistor cannot be turned 'off' and this forbids such device from being utilized as a digital signal processor, which is a key module in modern computer. Since the potential usefulness of graphene in electronic applications cannot be fully tapped unless a bandgap can be engineered, bandgap engineering of graphene has therefore becomes one of the most actively discussed topics. Several strategies have been suggested to achieve finite bandgap in graphene [11-16]. The most straightforward method being the introduction of an 'asymmetry' between the two sublattices in graphene unit cell. In this case, the onsite 'hopping' of electron at the two different sublattices differs by an energy amount of $\Delta$, and this creates a bandgap opening of $2 \Delta$. The broken sublattice symmetry can be induced by the semihydrogenation of graphene where hydrogen atoms are selectively attached to only one of the two sublattices [13-15]. Intrinsically, the spin-orbit-coupling in graphene also generates a small bandgap [16].

Recently, it has been shown that graphene and several graphene based structure exhibits exceptionally strong optical nonlinearity especially in the important terahertz frequency regime [17-20]. For gapless single layer graphene and bilayer graphene, the nonlinear optical response can easily dominates over the linear response when very moderate electric field strength of $10^{3} \sim 10^{4} \mathrm{~V} / \mathrm{cm}$ is applied [17-19]. The interband nonlinear optical response of gapped graphene was reported in our previous work [20]. In gapped graphene, the nonlinear optical conductivities exhibits 'two colour' optical response, i.e. there exists two distinct absorption peaks in the optical spectrum. Interestingly, the absorption peak corresponds to 3-photon absorption is well separated from the linear response and this leads to the existence of a frequency regime where the optical response is solely made up of the nonlinear component. In this work, we investigate the intraband nonlinear optical response in gapped graphene in the terahertz regime. By directly decomposing the charge carrier dynamics into linear and nonlinear components, we calculate the optical response of gapped graphene under strong-and weak-field condition. Finite doped and gapped graphene is considered in this calculation since the practical implementation of gapped-graphene-based photo-mixers requires finite temperature information and finite doping is inevitably present in most of the sample due to crystal imperfection. Under weak-field condition, it is found that a moderate level of bandgap opening in the order of few tens of meV results in enhanced third-order nonlinear optical response. When the external field is sufficiently strong, the third order nonlinear optical response behaves rather differently and the optical spectrum becomes 
complicated. There exists two distinct hotspots at (i) low temperature large bandgap; and (ii) high temperature small bandgap. For the nonlinear response to dominate over the linear response, the critical electric field strength is ranges between $10^{4} \mathrm{~V} / \mathrm{cm}$ and $10^{5} \mathrm{~V} / \mathrm{cm}$. Our results suggest that the optical nonlinearity of gapped graphene is strongly gapped dependent and hence the level of bandgap opening has to be carefully chosen in order to optimize the nonlinear photon-mixing effect.

\section{Theory}

The Hamiltonian of gapped graphene is given as

$H=\left[\begin{array}{cc}-\Delta & v_{F} p_{+} \\ v_{F} p_{-} & \Delta\end{array}\right]$

where the Fermi velocity is $v_{F}=3 t a / 2 \hbar \approx 10^{6} \mathrm{~m} / \mathrm{s}, t \approx 3 \mathrm{eV}$ is the nearest neighbour hopping integral, $a \approx 0.142 \mathrm{~nm}$ is the carbon-carbon distance, $p_{ \pm}=p_{x} \pm i p_{y}$ and $\mp \Delta$ is the onsite energy difference in sublattice $A$ and $B$ respectively. The energy eigenvalue is given by $\varepsilon_{s}(k)=s \sqrt{v_{F}^{2} p^{2}+\Delta^{2}}$ where the conduction band and valence band are represented by the index $S= \pm 1$ respectively. The energy eigenvalue clearly indicates a bandgap opening of $2 \Delta$ at the Dirac point. In contrast to gapless graphene in which the quasiparticles around K-point are massless Dirac Fermions, the gapped graphene low energy quasiparticles behaves like a massive Dirac Fermions where $\Delta$ acts as a mass-like term. The velocity operator is given by $\widehat{v}=\partial H / \partial p$ and the corresponding expectation value can be written as $\vec{v}_{s}=\partial \varepsilon_{s} / \partial p$. For gapped graphene, the velocity can be written as

$\vec{v}_{s}^{(0)}=\frac{s v_{F}^{2} \vec{p}}{\sqrt{v_{F}^{2} p^{2}+\Delta^{2}}}$

Consider a time-dependent in-plane electric field in the form of

$$
\vec{E}(\vec{r}, t)=\sum_{\mu} \vec{E}_{\mu} \exp \left[i\left(\vec{q}_{\mu} \cdot \vec{r}-\omega_{\mu} t\right)\right]
$$


where $\vec{E}_{\mu}, \vec{q}_{\mu}$ and $\omega_{\mu}$ are the amplitude, wavevector and frequency of $\mu$-th wave of the electric field. Ignoring the weak magnetic component, the external field can be coupled to the quasiparticle dynamics by the substitution $\vec{p}=\vec{p}-e \vec{A}(\vec{r}, t)$ where $\vec{E}(\vec{r}, t)=-\partial \vec{A}(\vec{r}, t) / \partial t$ and $e$ is the electric charge. The externally perturbed quasiparticles acquire linear and nonlinear velocities which can be separated by performing a Taylor expansion with respect to the external field. Up to third-order in the external field, the linear and nonlinear field-dependent velocities can be written as

$$
\begin{aligned}
& \vec{v}_{s}^{(1)}=\frac{s v_{F}^{2}}{\sqrt{v_{F}^{2} p^{2}+\Delta^{2}}}\left[\vec{u}-\frac{v_{F}^{2} \vec{p} \cdot \vec{u}}{v_{F}^{2} p^{2}+\Delta^{2}} \vec{p}\right] \\
& \vec{v}_{s}^{(2)}=\frac{s v_{F}^{2}}{\sqrt{v_{F}^{2} p^{2}+\Delta^{2}}}\left[-\frac{v_{F}^{2} u^{2}}{v_{F}^{2} p^{2}+\Delta^{2}} \frac{\vec{p}}{2}+\frac{v_{F}^{2} \vec{p} \cdot \vec{u}}{v_{F}^{2} p^{2}+\Delta^{2}}\left(\frac{3 \vec{p}}{2}-\vec{u}\right)\right] \\
& \vec{v}_{s}^{(3)}=\frac{s v_{F}^{2}}{\sqrt{v_{F}^{2} p^{2}+\Delta^{2}}}\left[-\frac{v_{F}^{2} u^{2}}{v_{F}^{2} p^{2}+\Delta^{2}} \frac{\vec{u}}{2}+\frac{v_{F}^{2} \vec{p} \cdot \vec{u}}{v_{F}^{2} p^{2}+\Delta^{2}} \frac{3 u^{2} \vec{p}}{2}+\frac{3 \vec{u}}{2}\left(\frac{v_{F}^{2} \vec{p} \cdot \vec{u}}{v_{F}^{2} p^{2}+\Delta^{2}}\right)^{2}-\frac{5 \vec{p}}{2}\left(\frac{v_{F}^{2} \vec{p} \cdot \vec{u}}{v_{F}^{2} p^{2}+\Delta^{2}}\right)^{3}\right]
\end{aligned}
$$

where for simplicity we have denoted $\vec{u}=-e \vec{A}$, and $\vec{v}_{s}{ }^{(i)}$ is the $i$-th order velocity acquired by the quasiparticles in gapped graphene per spin and per valley degeneracy. The velocities in conduction band has the same magnitude as that of the valence band and the sign is reverse due to the perfect 'updown' symmetry of the gapped graphene band structure. The optical current is given as

$$
\vec{J}^{(i)}=e \sum_{s} \int d \vec{p} \vec{v}_{s}^{(i)} f\left(\varepsilon_{s}, \mu\right)
$$

where $f\left(\varepsilon_{s}, \mu\right)$ is the Fermi-Dirac distribution with chemical potential $\mu$. When performing the velocities expansions, it is assumed that $p>u$. This assumption is reasonable since the presence of bandgap and finite doping level of $\mu$ will always ensure that the charge carriers involves in the optical transition possess sufficiently large momentum.

\section{$3 \quad$ Gapped Graphene Photon Mixing Effect}

From Eq. (5), the linear current can be explicitly written as

$$
\vec{J}^{(1)}=\frac{e \vec{u} v_{F}^{2}}{(2 \pi \hbar)^{2}} \sum_{s} \int p d p d \phi \frac{f\left(\varepsilon_{s}, \mu\right)}{\sqrt{v_{F}^{2} p^{2}+\Delta^{2}}}\left[1-\frac{v_{F}^{2} p^{2} \cos ^{2} \phi}{v_{F}^{2} p^{2}+\Delta^{2}}\right]
$$


where $\phi$ is the angle between $\vec{p}$ and $\vec{u}$. The third order nonlinear optical current is given by

$$
\begin{aligned}
& \vec{J}^{(3)}=\frac{e \vec{u} v_{F}^{2}}{(2 \pi \hbar)^{2}} \sum_{s} \int p d p d \phi \frac{f\left(\varepsilon_{s}, \mu\right)}{\sqrt{v_{F}^{2} p^{2}+\Delta^{2}}}\left\{\frac { s v _ { F } ^ { 2 } } { \sqrt { v _ { F } ^ { 2 } p ^ { 2 } + \Delta ^ { 2 } } } \left[-\frac{v_{F}^{2} u^{2}}{v_{F}^{2} p^{2}+\Delta^{2}} \frac{\vec{u}}{2}\right.\right. \\
& \left.\left.+\frac{v_{F}^{2} \vec{p} \cdot \vec{u}}{v_{F}^{2} p^{2}+\Delta^{2}} \frac{3 u^{2} \vec{p}}{2}+\frac{3 \vec{u}}{2}\left(\frac{v_{F}^{2} \vec{p} \cdot \vec{u}}{v_{F}^{2} p^{2}+\Delta^{2}}\right)^{2}-\frac{5 \vec{p}}{2}\left(\frac{v_{F}^{2} \vec{p} \cdot \vec{u}}{v_{F}^{2} p^{2}+\Delta^{2}}\right)^{3}\right]\right\}
\end{aligned}
$$

Eq. (6) and Eq. (7) can be solved analytically at zero temperature since the Fermi-Dirac distribution becomes a simple step function at $\mathrm{T}=0 \mathrm{~K}$. The linear and third-order nonlinear optical responses at zero temperature are in a rather simple form of

$\left.\vec{J}^{(1)} T=0=-X \frac{i e^{2}}{\pi \hbar} \sum_{\mu} \vec{E}_{\mu} e^{i\left[\vec{q}_{\mu} \cdot \vec{r}-\omega_{\mu} t\right.}\right]$

and

$$
\vec{J}_{T=0}^{(3)}=-Y \frac{i s e^{4} v_{F}^{2}}{8 \pi \hbar^{2} \mu} \sum_{\mu \nu \xi} \frac{\varepsilon_{p h}}{\mu-\varepsilon_{p h}} \frac{\vec{E}_{\mu} \cdot \vec{E}_{v} \vec{E}_{\xi}}{\omega_{\mu} \omega_{\nu} \omega_{\xi}} e^{i\left[\left(\vec{q}_{\mu}+\vec{q}_{v}+\vec{q}_{\xi}\right) \cdot \vec{r}-\left(\omega_{\mu}+\omega_{v}+\omega_{\xi}\right) t\right.}
$$

where

$$
\begin{aligned}
& X=\frac{\tilde{\mu}}{\eta(\Delta)^{1 / 2}}-\frac{\tilde{\mu}-1}{\eta(\Delta, \omega)^{1 / 2}} \\
& Y=\frac{\tilde{\mu}}{3} \frac{\eta(2 \Delta, 3 \omega)}{\eta(\Delta, 3 \omega)^{5 / 2}}-\left(\frac{\tilde{\mu}}{3}-1\right) \frac{\eta(2 \Delta)}{\eta(\Delta)^{5 / 2}}
\end{aligned}
$$

where $\tilde{\mu}=\mu / \hbar \omega, \eta(a \Delta)=1+(a \Delta / \mu)^{2}$ and $\eta(a \Delta, b \omega)=1+[a \Delta /(\mu-b \hbar \omega)]^{2}$. It can be seen that the second-order velocity $\vec{v}_{s}^{(2)}$ leads to zero second-order nonlinear optical response as a signature of crystal inversion symmetry. It can be seen that when $\Delta=0, \mathrm{X}=\mathrm{Y}=1$, and hence the optical responses reduce to gapless case [21]. 
The third order nonlinear current is proportional to the third power of the external field $\propto E^{3}$ while the linear current $\propto E$. Due to this different external field dependence, it is convenient to define a critical electric field strength $E_{c}$ such that the nonlinear response becomes as strong as the linear response, i.e. $\left|\vec{J}^{(3)}\right|=\left|\vec{J}^{(1)}\right|$ when $E_{c}$ is applied. A strongly nonlinear material is expected to have a smaller value of $E_{c}$ since the nonlinear response is already intrinsically strong. The $E_{c}$ is therefore a convenient indicator of the optical nonlinearity. The critical field strength for gapped graphene is given as $E_{c}=\varepsilon_{\Delta} E_{0}$ where $E_{0}$ is the zero temperature critical field strength in gapless graphene and $\varepsilon_{\Delta}=\sqrt{X / Y}$ is a dimensionless parameter signifying the effect of bandgap $\Delta$ on the critical field strength. The gap dependence of $\varepsilon_{\Delta}$ is plotted in Fig. 1. For $\mu=60 \mathrm{meV}, \omega=1 \mathrm{THz}$ and an arbitrary value of $\Delta=20 \mathrm{meV}$, the linear conductivity in gapped graphene is enhanced by a factor of $\mathrm{X}=1.05$ while the nonlinear conductivity in gapped graphene is enhanced by a factor of $Y=1.24$. This slightly reduces the critical field by a factor of $\varepsilon_{\Delta}=0.92$, i.e. the third-order nonlinear response is enhanced by $8 \%$ in comparison to gapless graphene. Interestingly, low temperature enhancement of interband third-order optical nonlinearity is also observed in gapped graphene [20]. Our results hence suggest that the optical nonlinearity enhancement due to bandgap opening is universal to both inter- and intraband nonlinear optical processes. For very large $\Delta, \varepsilon_{\Delta}$ is greater than one, signifying degradation of the optical nonlinearity. This arises because the large bandgap destroys too many low lying states and severely degrade the nonlinear response.

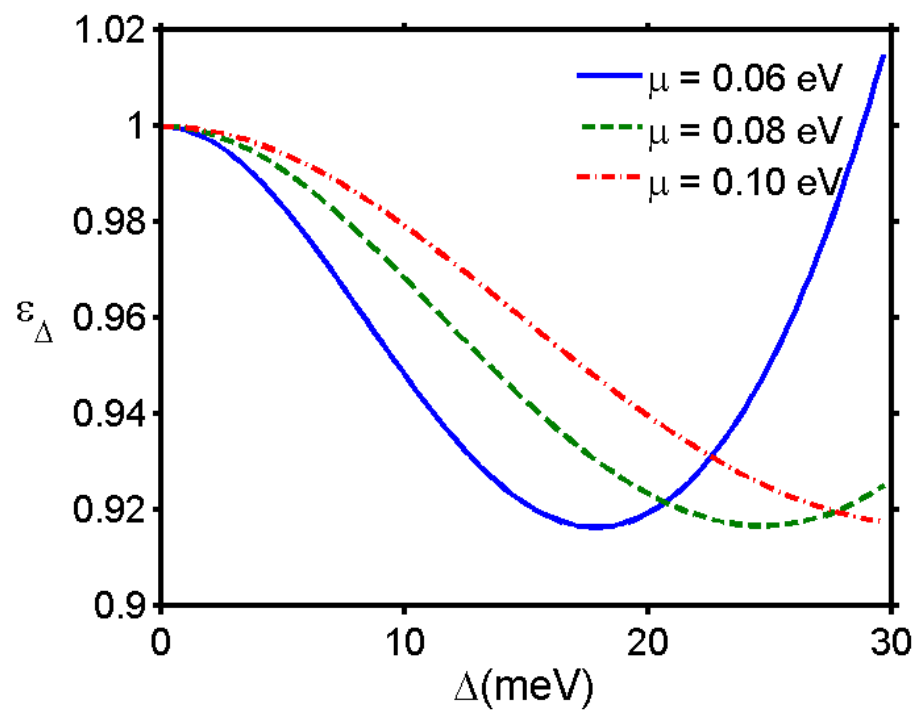

Fig. 1 - Gap dependence of $\varepsilon_{\Delta}$ at $\mathrm{T}=0 \mathrm{~K}$ and $\omega=1 \mathrm{THz}$. 
At finite temperature, the linear and nonlinear optical responses, as shown in Eq. (6) and Eq. (7), become rather complicated and have to be evaluated numerically. Since deep charge carriers cannot respond to the external field due to the unavailability of higher energy states, the lower limit of the momentum integration in Eq. (6) and Eq. (7) was chosen as $\mu-\varepsilon_{p h}-k_{B} T$. The momentum range of the excitable charge carrier is illustrated in Fig. 2. The temperature and gap dependences of gapped graphene are numerically plotted in Fig. 3. The following features can be observed: (i) a moderate value of bandgap opening enhances the nonlinear response at finite temperature; (ii) nonlinear optical response grows with increasing temperature; and (iii) in the large bandgap and high temperature regime (upper right corner of Fig. 3) the nonlinear response falls down rapidly and reverses the sign. Feature (i) is qualitatively the same as the zero-temperature critical field results mentioned above and hence it can be concluded that the enhanced nonlinear response at moderate level of bandgap opening is a generate feature at all temperature. For feature (ii), as temperature grows, deeper charge carriers can involve in the optical absorption due to the availability of higher thermally evacuated states. These carriers have lower momentum which results in the much larger nonlinear velocities as shown in Eq. (4). This eventually leads to the thermal enhancement of the nonlinear optical response.

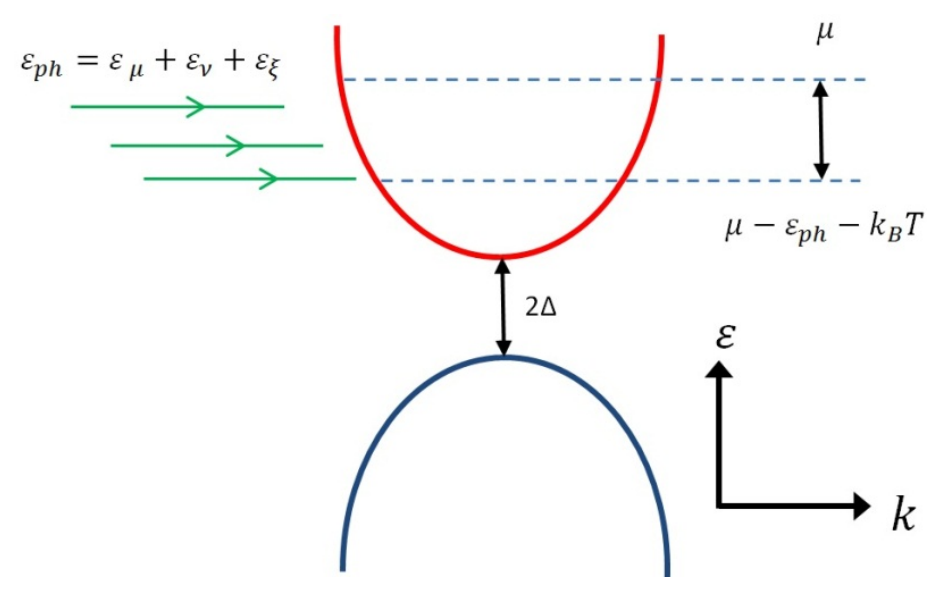

Fig. 2 - Schematic drawing of the gapped graphene band structure and the third order nonlinear optical process. At $\mathrm{T}=0 \mathrm{~K}$, the absorption of $\varepsilon_{p h}$ can only lead to the excitation of carriers staying between $\mu$ and $\mu-\varepsilon_{p h}$. At finite temperature, the lower-limit of the excitable carriers extend downward according to $\mu-\varepsilon_{p h}-k_{B} T$. Finite temperature Fermi-Dirac distribution extends beyond $\mu$ and is arbitrarily cut off at the upper limit of $\Lambda=0.5 \mathrm{eV}$ in the numerical calculation. 


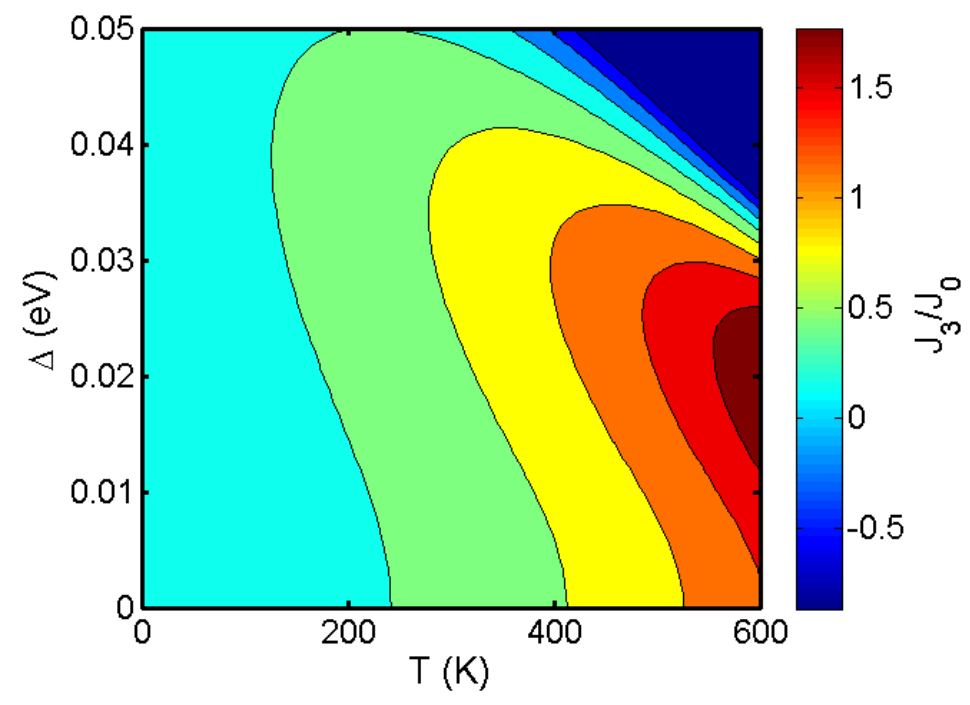

Fig. 3 - Third-order nonlinear optical conductivity at $\omega=1 \mathrm{THz}$ and $\mu=0.1 \mathrm{eV}$ under weak-field condition. The nonlinear current is normalized by $J_{0}=v_{F}^{2} e^{4} E^{3} / 8 \pi \hbar^{2}$.

\section{$4 \quad$ Strong-field Optical Response}

When the external field is sufficiently strong, the Fermi-Dirac distribution function in Eq. (5) with the unperturbed gapped graphene energy dispersion $\varepsilon_{s}(k)=s \sqrt{v_{F}^{2} p^{2}+\Delta^{2}}$ is no longer a reasonable assumption since the carrier energy should really be $\varepsilon=\varepsilon_{s}+\Delta \varepsilon_{s}$ where the additional fielddependent term $\Delta \varepsilon_{s}=\left(\vec{v}_{s}^{(1)}+\vec{v}_{s}^{(2)}+\vec{v}_{s}^{(3)}\right) \cdot \vec{p}$ is no longer negligible under strong-field condition. In this case, the carrier population redistribute themselves according to $f\left(\varepsilon_{s}+\Delta \varepsilon_{s}\right)=f_{0}+\Delta f_{1}+\Delta f_{2}+\Delta f_{3}$ where $f_{0}$ is the unperturbed Fermi-Dirac distribution function. We have performed an expansion third-order in terms of the external field on $f\left(\varepsilon_{s}+\Delta \varepsilon_{s}\right)$. Explicitly, the higher order expansions are written as:

$$
\begin{aligned}
& \Delta f_{1}=\left(\vec{v}_{s}^{(1)} \cdot \vec{p}\right) f_{0}^{(1)} \\
& \Delta f_{2}=\left(\vec{v}_{s}^{(2)} \cdot \vec{p}\right) f_{0}^{(1)}+\frac{\left(\vec{v}_{s}^{(1)} \cdot \vec{p}\right)^{2}}{2} f_{0}^{(2)} \\
& \Delta f_{3}=\left(\vec{v}_{s}^{(3)} \cdot \vec{p}\right) f_{0}^{(1)}+\left(\vec{v}_{s}^{(1)} \cdot \vec{p}\right)\left(\vec{v}_{s}^{(2)} \cdot \vec{p}\right) f_{0}^{(2)}+\frac{\left(\vec{v}_{s}^{(1)} \cdot \vec{p}\right)^{3}}{6} f_{0}^{(3)}
\end{aligned}
$$


where the n-th order derivative of the Fermi-Dirac distribution is denoted by $f^{(n)}=\partial f / \partial \varepsilon$. At $\mathrm{T}=0 \mathrm{~K}$, the strong-field linear and nonlinear response are given as

$$
\begin{aligned}
& \vec{J}_{T=0}^{(1 S)}=-S \frac{i e^{2}}{\pi \hbar} \sum_{\mu} \vec{E}_{\mu}\left[X+\Delta_{\mu} \Delta_{\omega}\left(1-\Delta_{\mu}^{2}\right)\right] e^{i\left[\vec{q}_{\mu} \cdot \vec{r}-\omega_{\mu} t\right]} \\
& \vec{J}_{T=0}^{(2 S)}=0 \\
& \vec{J}_{T=0}^{(3 S)}=-S \frac{i e^{4}}{8 \pi \hbar^{2} \mu} \sum_{\mu \nu \xi} \frac{\vec{E}_{\mu} \cdot \vec{E}_{\nu} \vec{E}_{\xi}}{\omega_{\mu} \omega_{\nu} \omega_{\xi}}\left[Y-\Delta_{\mu}^{2}+59 \Delta_{\mu}^{4}-177 \Delta_{\mu}^{6}+208 \Delta_{\mu}^{8}-90 \Delta_{\mu}^{10}\right] e^{i\left[\left(\vec{q}_{\mu}+\vec{q}_{\nu}+\vec{q}_{\xi}\right) \cdot \vec{r}-\left(\omega_{\mu}+\omega_{\nu}+\omega_{\xi}\right) t\right]}
\end{aligned}
$$

where the superscript 's' emphasizes the strong-field condition. The strong-field optical response of gapped graphene significantly differs from gapless graphene. In gapless graphene, both linear and second-order nonlinear optical responses are well protected from the strong-field effect. In gapped graphene, the bandgap opening alters the linear response and generates an additional gap-dependent term. The second-order nonlinear response remains zero in strong-field regime since the lattice inversion symmetry is retained. The finite temperature third-order nonlinear optical response in strongfield regime is numerically evaluated and is shown in Fig. 4. Under strong-field condition, the nonlinear response becomes rich in features. There exist two response 'hotspots' at: (i) low temperature with large bandgap; and at (ii) high temperature with small bandgap. These two hotspots are interconnected by a 'belt' of enhanced optical response. Hotspot (i) is also observed in the interband nonlinear conductivities of gapped graphene and this suggests that the low temperature gapenhancement of the optical nonlinearity in gapped graphene is universal to both interband and intraband processes in both weak-and strong-field regimes [21]. The 'hotspots' and the interconnecting 'belt' are due to the interplay between the thermal inclusions of more optically excitable charge carries at higher temperature and the level of bandgap opening. The thermal enhancement of the nonlinear current will stop when the downward extensions of the optically excitable carrier population reaches the conduction band edge, i.e. when $\mu-k_{B} T-\varepsilon_{p h}$ 'touches' the bandgap $\Delta$ since there are no more excitable charge carriers beyond the bandgap. Hotspot (ii) therefore occurs at small $\Delta$ and large temperature since it takes larger $k_{B} T$ for $\mu-k_{B} T-\varepsilon_{p h}$ to touch the smaller bandgap $\Delta$. This also explains the existence of hostpost (i) and the interconnecting 'belt'. 


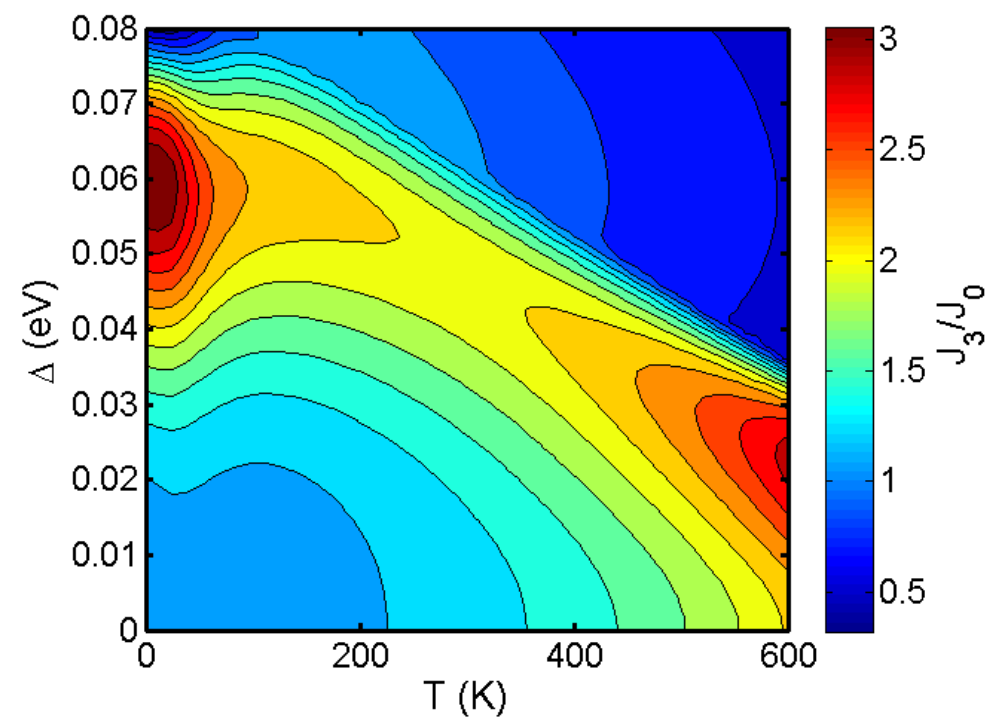

Fig. 4 - Third-order nonlinear optical conductivity at $\omega=1 \mathrm{THz}$ and $\mu=0.1 \mathrm{eV}$ under strong-field condition. There exists two response 'hotspot' at (i) low temperature large gap; and (ii) high temperature small gap regimes.

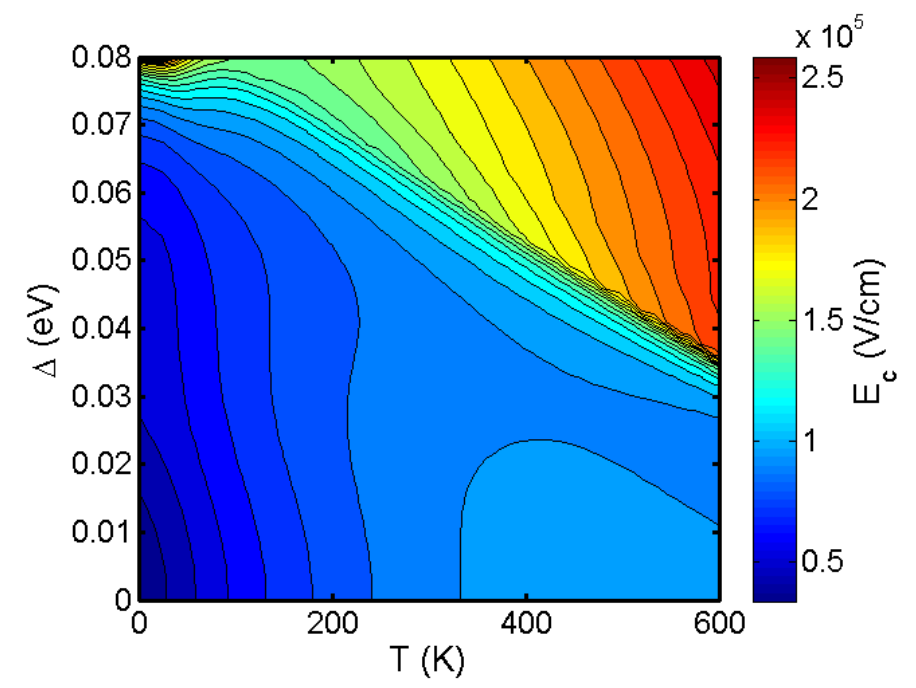

Fig. 5 - Critical electric field at $\omega=1 \mathrm{THz}$ and $\mu=0.1 \mathrm{eV}$ under strong-field condition. The critical field rises rapidly in the upper right regime where thermal inclusion of optically excitable carrier population reaches the conduction band edge.

The critical field is shown in Fig. 5. The critical field ranges between $10^{4} \sim 10^{5} \mathrm{~V} / \mathrm{cm}$ for various temperature and bandgap opening. This value is rather large in comparison to the gapless graphene since in gapped graphene both linear and nonlinear responses are amplified under strong-field condition, in contrast to gapless graphene where only nonlinear response is amplified while the linear 
response remains unchanged in strong-field regime. There is a sudden increase of the critical electric field in the upper right corner of Fig. 5. This happens when the nonlinear optical response stops increasing with the rising temperature as $\mu-k_{B} T-\varepsilon_{p h}$ touches the band edge (see Fig. 1). Beyond this temperature, the nonlinear response decreases dramatically since the charge carriers are thermally raised to higher energy states with larger momentum which, according to Eq. (4), carry much smaller nonlinear velocities. The third-order nonlinear optical response is a 3-photon process and it touches the band edge much earlier than the linear single-photon process. Therefore, at large bandgap opening or at high temperature regime, the nonlinear current diminishes while the linear response continues to grow. This leads to the sudden increase of the critical field and explains the occurrence of the triangularshaped high critical field regime as shown in Fig. 5.

\section{Conclusions}

In conclusion, we have investigated the intraband nonlinear optical response of gapped graphene with finite doping under both weak-and strong-field regimes. It is found that the optical response of gapped graphene depends strongly on the level of bandgap opening. For practical application of gapped graphene in nonlinear terahertz optoelectronics, the level of the bandgap opening and the operating temperature have to be carefully chosen such that the photon-mixing effect is optimized.

\section{Reference}

[1] K. S. Novoselov, A. K. Geim, S. V. Morozov, D. Jiang, Y. Zhang, S. V. Dubonos, I. V. Dubonos, I. V. Grigorieva, A. A. Firsov, Science 306, 666-669 (2004).

[2] A. K. Geim, K. S. Novoselov, Nature Mater. 6, 183-191 (2007).

[3] Y. Zhang, Y. Tan, H. L. Sormer, P. Kim, Nature (London) 438, 201-204 (2005).

[4] K. I. Bolotin, K. J. Sikes, Z. Jiang, M. Klima, G. Fudenberg, J. Hone, P. Kim, H. L. Stormer, Solid State Commun. 146, 351-355 (2008).

[5] A. B. Kuzmenko, E. van Heumen, F. Carbone, D. van der Marel, Phys. Rev. Lett. 100, 117401 (2008).

[6] C. Zhang, L. Chen, Z. Ma, Phys. Rev. B 77, 241402(R) (2008).

[7] V. P. Gusynin, S. G. Sharapov, Phys. Rev. Lett. 95, 146801 (2005).

[8] K. S. Novoselov, Z. Jiang, Y. Zhang, S. V. Morozov, H. L. Stormer, U. Zeitler, J. C. Maan, G. S. Boebinger, P. Kim, A. K. Geim, Science 315, 1379 (2007). 
[9] S. V. Morozov, K. S. Novoselov, M. I. Katsnelson, F. Schedin, L. A. Ponomarenko, D. Jiang, A. K. Geim, Phys. Rev. Lett. 97, 016801 (2006).

[10] K. S. Novoselov, A. K. Geim, S. V. Morozov, D. Jiang, M. I. Katsnelson, I. V. Grigorieva, S. V. Dubonos, A. A. Firsov, Nature (London) 438, 197-200 (2005).

[11] J. O. Sofo, A. S. Chaudhari, G. D. Barber, Phys. Rev. B 75, 153401 (2007).

[12] D. C. Elias, R. R. Nair, T. M. G. Mohiuddin, S. V. Morozov, P. Blake, M. O. Halsall, A. C. Ferrari, D. W. Boukhvalov, M. I. Katsnelson, A. K. Geim, K. S. Novoselov, Science 30, 610-613 (2009).

[13] G. Giovannetti, P. A. Khomyakov, G. Brocks, P. J. Kelly,J. van den Brink, Phys. Rev. B 76, 073103 (2007).

[14]. S. Y. Zhou, G. H. Gweon, A. V. Fedorov, P. N. First, W. A. de Heer, D. H. Lee, F. Guinea, A. H. Castro Neto, A. Lanzara, Nature Mater. 6, 770-775 (2007).

[15] L. Chen, Z. Ma, C. Zhang, Appl. Phys. Lett. 96, 023107 (2010).

[16] C. L. Kane, E. J. Mele, Phys. Rev. Lett. 95, 226801 (2005).

[17] A. R. Wright, X. G. Xu, J. C. Cao, C. Zhang, Appl. Phys. Lett. 95, 072101 (2009).

[18] S. A. Mikhailov, Europhys. Lett. 79, 27002 (2007).

[19] Y. S. Ang, S. Sultan, C. Zhang, Appl. Phys. Lett. 97, 243110 (2010).

[20] Y. S. Ang, C. Zhang, Appl. Phys. Lett. 98, 042107 (2011).

[21] S. Sultan, Y. S. Ang, C. Zhang, J. Opt. Soc. Am. B 29, 274 - 279 (2012) . 